\title{
CONCEPTUAL DESIGN IN A HIGH-TECH ENVIRONMENT
}

\author{
G. Maarten Bonnema, Fred J.A.M. van Houten \\ University of Twente, Department of Engineering Technology; \\ Laboratory of Design, Production and Management \\ P.O. Box 217, NL-7500 AE Enschede, The Netherlands \\ Phone: +31534892548,Fax:+31534893631g.m.bonnema@utwente.nl
}

\begin{abstract}
This article will give an overview over design process models before concentrating on the main subject: Conceptual Design, which has had less academic attention than the detail design phases. In high-tech environments specific conditions apply. This article will deal with these conditions.

Some views on design in general are treated and attention is paid to the conditions and steps and to the level of abstraction, goals, people and skills. The paper identifies the crucial role of the systems engineer in the conceptual design phase.

The conceptual design tasks and their results will be treated. As the conceptual designer is best aware of the overall functionality of the system and the interrelations, he is able to safeguard the concepts in later design phases and to make a good decomposition into subsystems.

The paper ends with conclusions and directions for Future Work.
\end{abstract}

Key words: conceptual design, support system, systems engineering, models

\section{INTRODUCTION}

To be competitive in a high-tech market, a company has to bring out advanced products in short time and with minimal effort. Thus, the design process has to be quick, yet effective. One way to achieve this is to spend more effort in the early phase of the process, the conceptual phase, to ensure a proper concept and feasibility. 
This article will explore the Conceptual Design Phase in high-tech environments after first giving a brief overview over the design process. Aspects that will be treated are among others: the level of abstraction, goals, starting points, people and skills involved and the models used. Also, attention will be paid to the links between systems engineering and conceptual design. The information is based both on literature and practical experience of the authors.

Sections $\underline{4}$ and $\underline{5}$ present the conclusions and plans for future work.

\section{DESIGN PROCESS}

The Design Process has been researched by many scientists like Pahl and Beitz [Pahl1996], Van den Kroonenberg, and Young and Allen. In [Pahl1996] and [Lutte2001] several models are described and compared.

\subsection{Conditions and Steps in Designing}

Before looking at one of those models, it is wise to discuss the essence of design. Figure 1 shows one view on design: The goal is to find resolutions to issues. The right side of the loop shows the steps to be taken; the left side shows conditions to these steps. The first condition is that issues can be addressed (recognised). This requires a mind-set not many people have by nature. However, some people have the right mindset but fail further in the loop. This creates Chindogu: inventions that "almost serve a useful purpose" [Kawakami, Chindogu].

The second step involves the development of concepts. Note that this is mentioned as a condition. So the designer has to be enabled to develop concepts. This raises the following questions as to how to develop concepts:

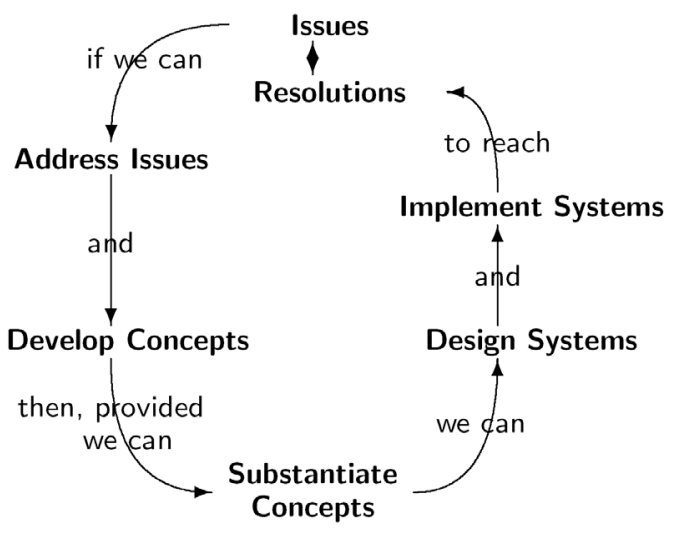

Figure 1: The Systems Design Process according to [Hitch1992].

- What is a concept and what characterises a good concept?

- How is a concept generated, or: What are the steps conceptual designers take to come to a proper concept?

- How many concepts are needed before choosing one? 
The developed concepts have to be substantiated in order to be of use. Thus a concept is only valid when its feasibility has been shown. This is hard to guarantee early in the design process. These issues will be addressed in Section $\underline{3}$.

When the conditions mentioned above are met, the design can be detailed and implemented. With a proper design and successful verification and testing, the issue under consideration has been resolved.

\subsection{Level of Abstraction}

To be able to focus on the conceptual phase, it is important to explore the relationship of the designer with his problem. In [Alexa1964] three situations are described (see Figure 2).

Design in an unselfconscious situation (Figure 2 a) acts directly on the artefact to be designed. The designer is the same as the workman that makes the artefact and the same as the end-user; ensuring optimal fit between context and form: any misfit is detected, resolved and implemented by one person playing three roles.

In a selfconscious situation (Figure $\underline{2}$ ) the designer is a specialist in the field of designing a specific class of artefacts (e.g. architects design

Context Form a. $\mathrm{C} 1 \longrightarrow \mathrm{F} 1$ actual world
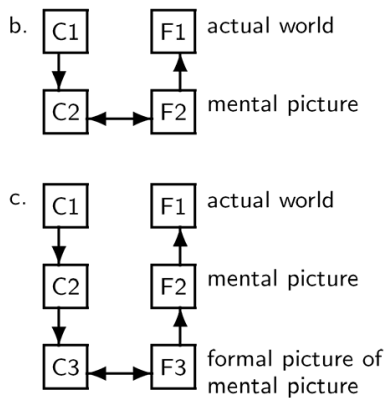

Figure 2: Three design situations [Alexa1964].

houses). However, as he has only limited experience in producing and using these artefacts, the design process runs via a conceptual picture of the environment and of a solution. This conceptual solution is then translated into a form (a product). As this is normally done by someone else than the designer or the user, the fit will be worse.

If the problem is more complex as is generally the case in high tech environments, the process will run via conceptual pictures and formal models of both the context and the form (Figure $2 \mathrm{c}$ ).

As can be seen, the distance between reality and the level of abstraction at which a solution is sought, and the number of translations increase with increasing complexity of the problem. According to [실. 1964], this results in worse fit of the artefact to the context (also see [Sage2000]).

The solution that is proposed in [Alexa1964] is to explore the extensive and as complete as possible list of requirements systematically by arranging the requirements in a tree-like structure based on the effect it has on a variable. Alexander believes that in this way the designer is directed to a solution. However, how a solution has to be found is left untreated. 
In [Schön1996] this problem is addressed as well:

A designer makes things. ... A system is complex in the specific sense that, whenever I make a move, I get results that are not just the ones that I intend. ... This unpredictability is a central attribute of design ...

The view presented in Figure $\underline{2}$ shows the gap many designers face between conceptual idea and realisation. It would therefore be an improvement when the behaviour of a possible realisation could be shown from the information in a concept.

\subsection{Model of Design}

Many of the design theories try to solve this problem. One model of the design process has been proposed in a classic book on conceptual design [Frenc1985], see Figure 3. Here, a scheme can be understood as a concept.

Although the steps are shown as distinct blocks, in reality they overlap. This is important to note, in particular in concurrent engineering; a strategy often used in high-tech environments.

The Need in this model is to be compared with the Addressed Issues in Figure $\underline{1}$. In Figure $\underline{3}$ the need is taken as the input to the design process. Since this paper will concentrate on conceptual design, it will take the need as an input as well.

Of course it should be noted that the description of the need as indicated by Marketing will, in many cases, be insufficient for designers. Elaboration is required in order to be able to design the needed product. This is indicated by the block Analysis of Problem; the result of which is a Statement of Problem. The blocks Conceptual Design and Selected Schemes will be treated in Section $\underline{3}$.

It is worthwhile to give a citation from [Frenc1985] about the conceptual design phase:

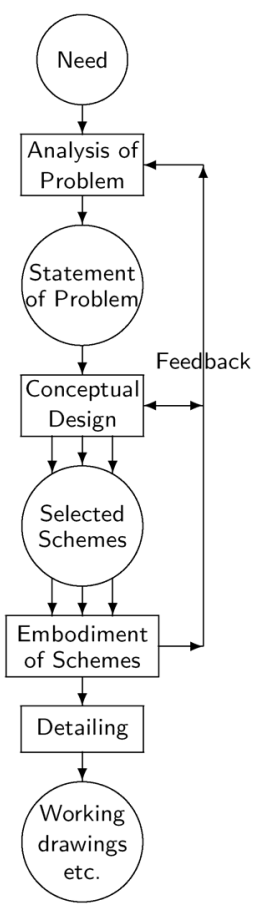

Figure 3: Design Process according to [Frenc1985].

It is the phase where engineering science, practical knowledge, production methods, and commercial aspects need to be brought together, and where the most important decisions are taken.

When a concept (or concepts) has been selected it has to be developed further in the Embodiment and Detailing phases, resulting in a set of 


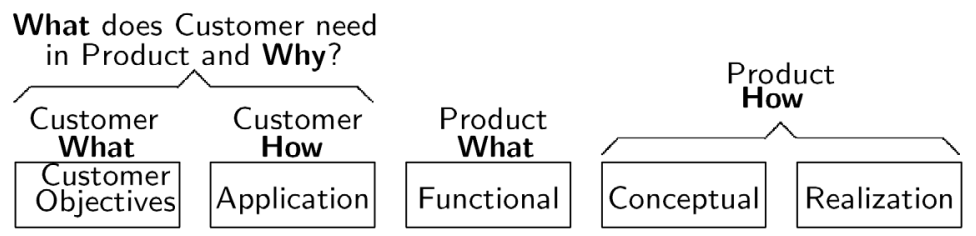

Figure 4: Basic CAFCR Model as presented in [Mulle2002].

drawings, a CAD-model, a production plan, facilities layout etc. This phasing is also often present in other models of the design process. The model by French will be used here as a reference for the design process. More models and a comparison can be found in [Pahl1996, Lutte2001].

In [Mulle2002] an interesting view on the creation of an architecture (the framework wherein the designer has to find a concept) of a system is shown: The Customer Objectives, Application, Functional, Conceptual, Realisation model, short: CAFCR-model (Figure 4). It can be used to describe the views on a system in relation to its context. This model is therefore a step in the direction of solving the problem shown in Figure 2 .

In Figure 2 $\mathrm{c}$ the route from context to form runs via mental and formal pictures. The content of these pictures is shown in Figure 4 : the two lefthand blocks depict the mental and formal pictures of the context. The two right-hand blocks describe the formal (conceptual) and mental (realisation) picture of the solution. The functional block describes what the product does.

In Table 1 the emphasis on the architectural aspects of the CAFCRmodel in each of the phases of the design process is shown. Note the diagonal shape of the table and that the conceptual phase is the only phase that covers all aspects of the CAFCR-model.

Table 1. Architectural aspects in design in different design phases. The number of +-es shows the importance of the aspect in the corresponding design phase. (Also see Figure $\underline{3}$ and $\underline{4}$.)

\begin{tabular}{lccccc}
\hline & $\begin{array}{c}\text { Customer } \\
\text { Objectives }\end{array}$ & Application & Functional & Conceptual & Realisation \\
\hline Need & ++ & + & & & \\
\hline Analysis of Problem & ++ & ++ & + & & \\
\hline Conceptual Design & + & ++ & ++ & ++ & + \\
\hline Embodiment & & + & + & + & ++ \\
\hline Detailing & & & & & ++ \\
\hline
\end{tabular}

In [Suh1990] axiomatic design is introduced. This design method is based on two axioms:

1. Maintain the independence of functional requirements.

2. Minimize the information content.

These axioms should guide the design process. However, in [Suh1990] it is also said that design consists of two processes: the creative and the analytical process. The creative process creates new solutions; the analytical 
process evaluates the new solutions and forces decisions. Axiomatic design is proposed as useful in the analytical phase, not in the creative phase.

The conclusion from the above is that there are many views on the design process and the way a designer faces a problem. An experienced designer will often switch viewpoints in order to explore and clearly define the problem and search for innovative solutions. The way problems are perceived are treated, how they are solved is not dealt with in the models above.

\section{CONCEPTUAL DESIGN}

Designing complex systems, e.g. aircraft and wafer steppers, requires a large amount of effort, resources and time. It is essential to direct and concentrate the effort in one direction as soon as possible.

The goal of the conceptual design phase is to explore the problem and the field of solutions, find the best solution that is feasible, plan how this solution can be implemented, work it out to a stage that the whole team can start working on it, and hire and train the people needed.

As can be seen in Figure $\underline{3}$, conceptual design takes place early in the design process where only the need and the problem are stated. As the need and problem will become clearer while designing concepts any conceptual designer should include reviewing the need and requirements.

On the other side of the conceptual design phase in Figure $\underline{3}$, one can see the embodiment phase. As the conceptual designer is best aware of the essentials, drawbacks and characteristics of the chosen concept, he is the best person to guard the concept during the embodiment and detailing phases.

In [Krumh1974] the conceptual phase is ordered along three orthogonal axes: Concreteness (Physical principle $\rightarrow$ Operating principle $\rightarrow$ Construction element), Complexity (Unit $\rightarrow$ Chain $\rightarrow$ Structure), and Realisation (Idea $\rightarrow$ Product). The route of the conceptual design phase through the space created by these three axes is shown in Figure $\underline{5}$. Note that the starting point (List of Requirements) and the end point (Total Solution) are concrete. The design process, however, has to raise the abstraction level and lower the complexity before solutions can be sought, combined and selected.

But what is a concept, actually? In [Pahl1996] no definition is given. But from the detailed description of the conceptual design phase it is clear that essential elements are working principles, a basic structure and calculations.

[Frenc1985] does not use the word concept but uses the word scheme. The terms in italics in the next quotation describe the characteristics of a good concept (italics by the author): 


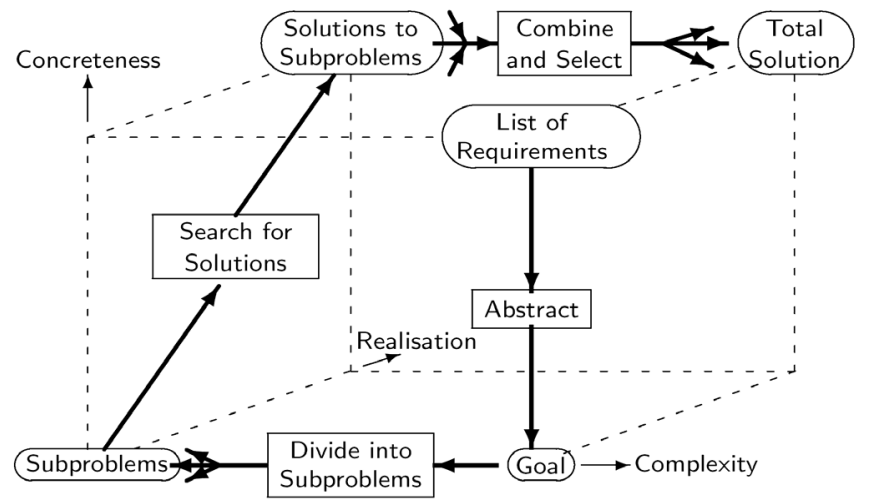

Figure 5: The space of the conceptual design phase [Krumh1974]. Ovals indicate information, rectangles show information processing.

By a scheme is meant an outline solution to a design problem, carried to the point where the means of performing each major function has been fixed, as have the spatial and structural relationships of the principal components. A scheme should be sufficiently worked out in detail for it to be possible to supply approximate costs, weights, and overall dimensions, and the feasibility should have been assured as far as circumstances allow.

From this it is clear that functions are very important. For each function a principle solution has to be found. The configuration of these principle solutions then provides the basic structure of the concept.

[Krumh1974] distinguishes physical and operating principles and construction elements. A physical principle is the basic physical effect that is used. An operating principle is a physical principle plus a form; a concrete object that uses this operating principle is called a construction element.

We will therefore use the following definitions:

Concept: A structured set of operating principles.

Operating principle: A physical principle with a possible instantiation.

Thus, creating concepts requires selecting operating principles and structuring them.

Having said this, do we need one, ten or hundreds of concepts before choosing one to proceed with? Hundreds makes it hard to identify the best one and is a waste of effort. With only one concept, there is nothing to choose. So between one and ten seems appropriate. Some four $( \pm 2)$ concepts can be overseen, can be made sufficiently different and can be worked out without wasting too much effort. When computer support for conceptual design is implemented, this number may increase, as less effort is required for creating and evaluating concepts. 
In many cases barriers in, for instance, technology have to be negotiated. It is well known that early in the design process not many costs are made, but the largest part of the costs to actually build the system are defined. Thus it is important to make the basic design decisions very carefully and as early as possible to focus the effort of all designers. Or: one concept has to be chosen as early as possible, but not sooner than when the consequences of the decision can be overseen. Choosing this moment is an issue in itself.

\subsection{People and Skills Involved}

In some cases the conceptual design can be made by one person but in high tech environments it is usually done by a team as there are usually several disciplines involved and many aspects to be considered. Although small compared to the team needed to develop the concept into a detailed product, it requires highly skilled people with different viewpoints. This Section will elaborate upon these people, their skills and roles.

From Figure $\underline{3}$ it can be seen that the conceptual design phase is based on the information in a statement of problem based on a need. The Marketing department usually states this need. It should therefore be involved to ensure the problem described by the designers is the correct problem.

It is important that the analysis is taken to a wide enough extent so that the core-problem is addressed. In some cases the need may even be resolved without any product. Though economically not interesting for the company it is in many cases the best solution.

If the problem is clearly defined and the essence is agreed upon by Marketing and the design department, technical designers have to find the best concept to solve it. Figure $\underline{6}$ shows the different designers and their knowledge-fields. All types of designers shown in Figure $\underline{6}$ may be needed in the conceptual design phase,

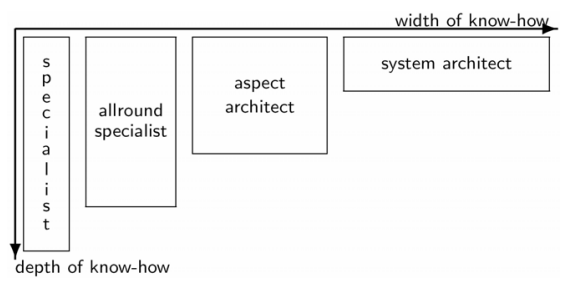

Figure 6: The width and depth of knowledge of different designers. Adapted from [Mulle2001] although the ones with the wider view (the aspect architects and system architects) will play the major role.

In the conceptual phase, the basic structure of the product is created. But also the production facilities and the process are to be considered in view of the total life cycle. Systems Engineering is vital in this. According to [Blanc1998] the following aspects are to be emphasised:

1. Improving methods for defining system requirements,

2. Addressing the total system from a life cycle perspective,

3. Considering the overall system hierarchy and interactions, 
4. Organising and integrating the necessary disciplines, and

5. Establishing a disciplined approach with reviews, evaluation and feedback.

According to [Sage2000] the systems engineering team acts as an interface team that provides the conceptual design and the technical direction.

From the description of the people involved, it can be concluded that the following skills are necessary in the team of conceptual designers: communication over disciplines, willing to learn, maintaining a clear view on the customer, documentation, having a wide perspective, and having an open mind.

\subsection{Tasks}

In Figure 7 the steps of the conceptual phase according to [Pahl1996] are shown. This process is fairly detailed. Functions play the most important role. These can be subdivided into sub-functions if necessary. It may be needed to introduce auxiliary functions. These perform actions not essential for the main function, but necessary for practical reasons (e.g. removal of waste material). It is also wise to distinguish supplementary functions which perform extra functions [Eekel1995]. Later on the focus shifts to finding suitable operating principles, and in the end to rating the found concepts.

[Pahl1996] advices the designer to abstract as much as possible. This can be done by omitting requirements on extras, stating the requirements as qualitatively

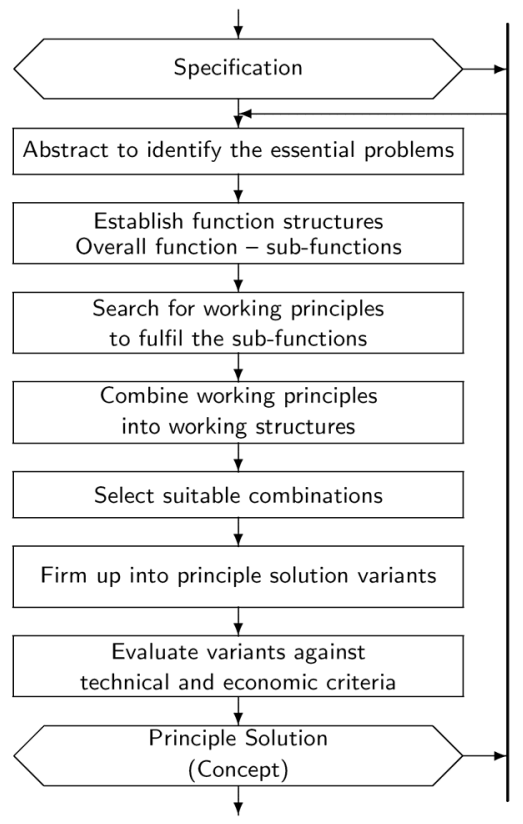

Figure 7: The Conceptual Design Phase according to [Pahl1996]. as possible and formulating the problem as neutrally as possible. This produces the goal of the design procedure.

The process shown in Figure $\underline{7}$ omits two tasks having a tight relation with systems engineering: documentation and division into subsystems and/or modules.

Documenting important decisions is paramount for guarding the concept later on in the process. 
The possibility to divide a concept into subsystems or modules is important in choosing the concept while some functions can only be performed properly by a system-wide approach. Thus dividing a system into subsystems consists of two actions: (1) mapping (sub)functions on (sub)systems (a one-on-one mapping will not always possible) and (2) carefully placing the interfaces. Performing these actions in unison can lead to a proper system architecture.

\subsection{Product Models in Conceptual Design}

When doing conceptual design, the designer will use many models to clarify his view on the problem, to experiment with solutions, to simulate solutions etc. One of the main uses of models is to communicate with fellow designers. This Subsection contains a concise inventory of models, their development, and the shift of focus. Also see Table 1 and [Ruiz2002].

In the early part of the conceptual design phase the requirements form the main model. This textual model is the starting point of conceptual design. When analysing the problem, the main form will remain text, however some sketches can aid in deciding what the exact problem is and may be used to draw a coarse outline of the product

As stated above, the functional view on the problem is in the early stages the most important. Thus functional diagrams are needed (e.g. state diagrams and FAST-ER in [Brace1996]). Schematics will become more important and coarse budgets for accuracy, energy consumption, space and weight have to be composed.

After this, principles to perform the functions have to be found. Here, pictures will become very important (iconic diagrams, bond graphs, blockdiagrams etc.). A morphological scheme or matrix ([Pahl1996]) may aid in this process. Also, more concrete product models may be used. For instance models that show the system with possible influences from manufacturing imperfections (e.g. tolerances) and the environment. The earlier mentioned budgets will be refined.

From here the diagrams will become more like technical drawings. The production facilities have to be developed in the detailing phase.

\section{CONCLUSIONS}

From the exploration of conceptual design above we can conclude that the results of the conceptual design phase determine the course of the design process of high-tech products to a very large extent. Based on descriptions of a need, the conceptual designers have to find a concept for a solution. First 
step is to define a goal. Then an architecture may be needed which serves as a basis for the concepts.

A concept can be defined as a structured set of operating principles; operating principles are defined as physical principles with a possible instantiation.

In general some four $( \pm 2)$ concepts have to be developed before choosing one. This number is based on the fact that ten or more seems a waste of effort and if there is only one there is nothing to choose and compare. So depending on the maturity of the field, two to six concepts are required. Using computer support a larger number of concepts can be useful as both creation and selection of concepts is made easier.

To come to concepts, many different (product) models will be used. Lists of requirements, interface specifications, sketches of principles may all be used. There exist many connections between them; changing one model has effect on most of the other ones. Using the models, concepts are developed using experience, patent searches, prior art, analyses, creative processes, etc.

In the conceptual phase there are many activities. A support system for this phase must be helpful in those aspects that are difficult or require a lot of work.

\section{FUTURE WORK}

The aim of future research is to design and implement a support system for conceptual design. This support system should reduce the gap between conceptual idea and realisation. Also it can help in making careful and welldocumented concept decisions. In the later phases of the design process the conceptual designer(s) can monitor detail decisions and verify the proper implementation of the chosen concept, thus preventing major modifications in the prototype and test phases.

To achieve this, the first steps to be taken are:

- Further investigate available methods and tools for Conceptual Design.

- Make an inventory of models used in Conceptual Design, including relations and translations between them.

- Develop methods for investigating behaviour of concepts, like qualitative simulation and parameter sensitivity simulation.

- Investigate the go/no-go decision making in concept selection. 


\section{REFERENCES}

[Alexa1964] Alexander, Christopher; Notes on the Synthesis of Form; Harvard University Press, Cambridge, Massachusetts ISBN: 0-674-62750-4

[Blanc1998] Blanchard, Benjamin S.; Fabrycky, Wolter J.; Systems Engineering and Analysis; Prentice Hall, ISBN 0-13-135047-1

[Brace1996] Bracewell, R.H.; Sharpe, J.E.E; Functional Descriptions used in Computer Support for Qualitative Scheme...; AI EDAM Journal - Special Issue: Representing Functionality in Design Vol.10, No.4; pp. 333-346

[Chindogu] Chindogu website; http://website.lineone.net/ sobriety/

[Eekel1995] Eekels, J.; Poelman, W.A.; Industriële productontwikkeling, Deel 2: Methodologie; Lemma, Utrecht (in Dutch); ISBN: 90-5189-435-X

[Frenc1985] French, Michael; Conceptual Design for Engineers; The Design Council London; Springer-Verlag; ISBN: 0-85072-155-5

[Hitch1992] Hitchins, Derek K.; Putting Systems To Work; J. Wiley and Sons, Chichester, UK; http://www.hitchins.co.uk/e-Putting\%20SystemsToWork.pdf

[Kawakami] Kawakami, Kenji; 101 Unuseless Japanese Inventions; HarperCollins, London.

[Krumh1974] Krumhauer, Peter; Rechnerunterstützung für die Konzeptphase der Konstruktion; Dissertation TU Berlin, 1974, D83 (in German)

[Lutte2001] Lutters, D.; Manufacturing Integration Based on Information Management; Ph.D. Thesis, University of Twente, 2001, ISBN 90-365-1583-1

[Mulle2001] Muller, Gerrit; The Arisal of a System Architect; Philips Research; http://www.extra.research.philips.com/natlab/sysarch/MaturityPaper.pdf

[Mulle2002] Muller, Gerrit; Short Introduction to Basic "CAFCR" Model; http://www.extra.research.philips.com/natlab/sysarch/BasicCAFCRPaper.pdf

[Suh1990] Suh, Nam P.; The Principles of Design; Oxford University Press New York Oxford, ISBN: 0-19-504345-6

[Pahl1996] Pahl, G.; Beitz, W.; Engineering Design - A Systematic Approach; SpringerVerlag London, ISBN: 3-540-19917-9

[Ruiz2002] Ruiz Zwollo, Cristobal; Computer Aided Conceptual Design and Embodiment Generation; Master Thesis; University of Twente

[Sage2000] Sage, Andrew P.; Armstrong, James E. jr.; Introduction to Systems Engineering; Wiley Series in Systems Engineering; John Wiley and Sons, Inc.; ISBN 0-471-02766-9

[Schön1996] Schön, Donald; Bennet, John; Reflective Conversation with Materials; Bringing Design to Software; Winograd, Terry (ed.); Addison-Wesley, ISBN 0-201-85491-0 